\title{
Atuação do fisioterapeuta nos núcleos de apoio à saúde da família em Teresina, Piauí
}

\author{
Physiotherapeutic performance in the family \\ health support centers in Teresina, Piauí
}

\author{
Allan Dellon da Silva' ${ }^{1}$ \\ Luciana Tolstenko Nogueira2 ${ }^{2}$ (1) \\ Hengrid Graciely Nascimento Silva ${ }^{3}$ (1) \\ Samanta Cris Monteiro Frota ${ }^{4}$ (1)
}

1'Autor para correspondência. Universidade Estadual do Piauí (Teresina). Piauí, Brasil. dellonallan09@gmail.com

2-4Universidade Estadual do Piauí (Teresina). Piauí, Brasil. lutolstenko@hotmail.com, hengrid_graciely@hotmail.com, samantafrota25@gmail.com

RESUMO | INTRODUÇão: A atuação do fisioterapeuta vai muito além do reabilitar, pois este está inserido em todo o processo de produção de cuidado, dessa forma, desempenha um importante papel na atenção básica. OBJETIVO: Analisar a atuação do fisioterapeuta nos Núcleos de Apoio à Saúde da Família em Teresina, Piauí. MATERIAIS E MÉTODOS: Trata-se de um estudo descritivo, com delineamento transversal e abordagem quantitativa. Com aprovação pelo Comitê de Ética em Pesquisa, a coleta de dados se deu por meio da aplicação de um questionário semiestruturado com todos os fisioterapeutas que trabalham no NASF-AB de Teresina-PI. RESULTADOS: Todos os profissionais realizam atendimentos individuais na Unidade Básica de Saúde, e as disfunções mais comumente encontradas estão relacionadas às áreas de Traumato-ortopedia (100\%), neurologia $(83,33 \%)$, reumatologia (50\%) e fisioterapia respiratória (33,33\%). A maioria dos fisioterapeutas realizam atividades em grupo, ações preventivas e educação em saúde voltada para saúde da criança e adolescentes, saúde da mulher, saúde do homem e saúde do idoso. 100\% dos profissionais afirmaram realizar acompanhamentos domiciliares, dentre as atividades mais comuns destacam-se as orientações para pacientes e familiares, prescrição de dispositivos auxiliares de marcha e prescrição de exercícios domiciliares. Além disso, a maioria dos participantes mostrou-se insatisfeito quanto à interdisciplinaridade e interação entre as equipes. CONCLUSÃo: Conclui-se que os profissionais realizam atendimentos tanto individual, como de forma coletiva e os atendimentos ocorrem tanto na unidade básica de saúde como no domicilio. No entanto, ainda é comum relacionarem suas atividades à prática clinica e assistencialista.

PALAVRAS-CHAVE: Fisioterapia. Atenção primária à saúde. Promoção da saúde.
ABSTRACT | INTRODUCTION: The physiotherapist's performance goes far beyond rehabilitation, as this is inserted in the entire care production process, thus, it plays an important role in primary care. OBJECTIVE: To analyze the role of the physiotherapist in the Family Health Support Centers in Teresina, Piauí. MATERIALS AND METHODS: This is a descriptive study, with a cross-sectional design and quantitative approach. With approval by the Research Ethics Committee, data collection took place through the application of a semi-structured questionnaire with all physical therapists who work at NASF-AB in Teresina-PI. RESULTS: All professionals perform individual care at the Basic Health Unit, and the most commonly found disorders are related to the areas of Traumato-orthopedics (100\%), neurology (83.33\%), rheumatology (50\%) and respiratory physiotherapy (33.33\%). Most physical therapists perform group activities, preventive actions and health education focused on the health of children and adolescents, women's health, men's health and the health of the elderly. $100 \%$ of the professionals stated that they carry out home follow-ups. Among the most common activities, guidelines for patients and family members, prescription of auxiliary walking devices and prescription of home exercises stand out. In addition, most participants were dissatisfied with the interdisciplinarity and interaction between the teams. CONCLUSION: It is concluded that the professionals provide care both individually and collectively and the care takes place both in the basic health unit and at home. However, it is still common to relate their activities to clinical and care practice.

KEYWORDS: Physiotherapy. Primary Health Care. Health promotion. 


\section{Introdução}

Visando melhorias na assistência e garantia do direito à saúde de forma integral à população, em 1994 o Ministério da Saúde criou o Programa Saúde da Família (PSF), que posteriormente, com a expansão do programa e a proposta de reorganização da Atenção Básica no Brasil, passou a ser chamado de Estratégia Saúde da Família (ESF)ํ․․

Nessa perspectiva, com o intuito de fortalecer a rede de atenção básica e melhorar a qualidade da assistência, mediante a Portaria GM n ${ }^{\circ} 154$, de 24 de janeiro de 2008, foi criado o Núcleo de Apoio à Saúde da Família (NASF), que é o programa no qual a fisioterapia pode estar inserida para atuação na atenção básica, cujo principal objetivo é apoiar as equipes do ESF ${ }^{2-4}$. Recentemente foi aprovada a nova Política Nacional de Atenção Básica à Saúde (PNAB), que apesar de não haver grandes mudanças estruturais no NASF, houve mudança na nomenclatura, que passou a ser denominada de Núcleo Ampliado de Saúde da Família e Atenção Básica (NASF-AB) ${ }^{5}$.

O NASF-AB é constituído de três a cinco profissionais para cada equipe de ESF, sendo que a escolha desses profissionais é de responsabilidade do gestor local, de acordo com as necessidades da população. Esses profissionais possuem sua atuação pautada no atendimento integral e voltada às demandas individuais e coletivas de atenção à saúde ${ }^{5}$. Além disso, o profissional deve nortear sua prática nos princípios básicos preconizados pelo programa, a fim de garantir melhor assistência aos usuários, maior articulação entre as equipes do ESF, autonomia dos indivíduos e coletivos, e consequentemente melhor efetividade nos serviços ${ }^{6,7}$.

Historicamente, a fisioterapia era vista como uma profissão estritamente reabilitadora, pois se originou como uma profissão vinculada exclusivamente ao campo da reabilitação, na qual objetivava, sobretudo, a reabilitação de pessoas com lesões físicas resultante de guerras, e essa ideia de modelo curativista perdurou por alguns anos 2 . Todavia, essa concepção tem mudado ao longo dos anos, visto que para corresponder às mudanças no contexto de assistência à saúde e a lógica de organização do Sistema Único de Saúde (SUS), a necessidade por esses profissionais na atenção básica é crescente, além disso, a contribuição dessa categoria para a saúde coletiva tem despertado um novo olhar acerca da sua atuação ${ }^{8}$.

A atuação do fisioterapeuta vai muito além do reabilitar, pois este está inserido em todo o processo de produção de cuidado, dessa forma, desempenha um importante papel na atenção básica. A inserção desse profissional na equipe de saúde deve ser vista como uma estratégia de fortalecer esse nível de atenção, contribuindo para uma maior resolutividade e garantia da assistência de forma integral? Dentre as inúmeras atribuições da fisioterapia na atenção básica destacam-se a identificação da presença de distúrbios cinético-funcionais, orientações posturais, incentivo à participação comunitária em questões relacionadas à saúde, educação permanente, bem como orientações quanto a ambientes e estilo de vida saudáveis ${ }^{10}$.

No geral, os profissionais que compõem a equipe do NASF-AB ainda vivenciam alguns obstáculos que dificultam o processo de trabalho, e consequentemente uma atuação mais efetiva. Dentre eles, há a dificuldade para implementação de ações mais efetivas no que diz respeito à prevenção de doenças e promoção da saúde, além da escassez na prática de planejamento dessas ações, resistência por parte dos profissionais ao modelo de apoio matricial, e persistência da lógica de atuação ambulatorial ${ }^{11}$. Associado a isso, um complicador que ainda é relatado por profissionais que vivenciam essa realidade é a falta de capacitação para atuação nesse campo, bem como a falta de recursos para o desenvolvimento de atividades e infraestrutura física adequada ${ }^{3}$.

Nesse sentido, conhecer a realidade de atuação desses profissionais, assim como as facilidades e desafios frente à atuação na atenção básica é uma proposta viável, visto que poderá fornecer dados que possam identificar se as propostas e diretrizes preconizadas pelo NASF-AB estão de fato sendo desenvolvidas, e dessa forma contribuir para um melhor planejamento das atividades desenvolvidas por esses profissionais, bem como servir de comparativo para outras regiões do país, nas quais o programa esteja em execução. Diante disso, esse estudo objetivou analisar a atuação do fisioterapeuta nos Núcleos de Apoio à Saúde da Família em Teresina, Piauí. 


\section{Materiais e métodos}

Trata-se de um estudo descritivo, com delineamento transversal e abordagem quantitativa, que teve como sujeitos todos os fisioterapeutas vinculados às equipes do Núcleo de Apoio à Saúde da Família, localizados na cidade de Teresina (PI).

Para a confirmação da quantidade amostral foi realizada uma visita à Fundação Municipal de Saúde do município estudado, a fim de verificar o número de NASF-AB ativos na cidade e dentre estes, quais possuem o fisioterapeuta como integrante da equipe. Atualmente, a cidade de Teresina possui 03 NASF$A B$, que dão assistência à 26 Equipes de Saúde da Família, sendo que para cada equipe do NASF-AB há a presença de dois fisioterapeutas, e o vínculo empregatício desses profissionais se dá por meio de concurso público ${ }^{12}$.

Uma das limitações metodológicas deste estudo refere-se ao tamanho da população estudada para um estudo transversal, entretanto como mencionado anteriormente o cenário do estudo só dispunha desses profissionais.

A coleta de dados ocorreu no período compreendido entre novembro de 2018 a fevereiro de 2019. Para este fim, realizou-se contato prévio com os 06 fisioterapeutas vinculados às três equipes do NASF-AB existentes em Teresina-PI, cadastradas na Fundação Municipal de Saúde, foi agendado horário e local para exposição dos objetivos da pesquisa, bem como de riscos e benefícios, em seguida, foi acordada a coleta de dados, por meio da aplicação de um questionário semiestruturado, adaptado dos estudos de Candido ${ }^{13}$ e Sousa ${ }^{14}$, todos os participantes optaram por responder o questionário na Unidade Básica de Saúde (UBS) a qual estavam em atividade no dia agendado, e os questionários foram imediatamente respondidos e entregues ao pesquisador responsável.

O questionário foi organizado e dividido em seções, no qual na primeira seção foram solicitadas informações para caracterização da amostra, como sexo, idade, tempo de atuação no NASF-AB e qualificações, sem identificação do participante, a partir da segunda seção o questionário é constituído por perguntas acerca de atendimento individual na unidade de saúde, atendimento domiciliar, e perguntas sobre a realização de atividades voltadas exclusivamente para a saúde da criança e do adolescente, saúde da mulher, saúde do homem e saúde do idoso. E a última seção do questionário visou avaliar a satisfação dos profissionais quanto as condições de trabalho, no que diz respeito à infraestrutura e ambiência, e interação entre as equipes.

Outra limitação metodológica que pode ser apontada é em relação ao uso de um instrumento de coleta adaptado, no entanto optou-se por esse questionário adaptado pelo fato de abranger todos os grupos que teoricamente devem ser assistidos pelo fisioterapeuta na atenção básica, bem como avaliar a atuação do profissional nesse nível de atenção, logo acredita-se que permitiu apreender as questões relevantes do contexto pesquisado a que o estudo se propõe.

Para avaliação estatística dos dados coletados a análise se deu de forma descritiva, no qual o conteúdo do questionário foi organizado e tabulado por meio do Software Microsoft Excel e do software Statistical Package for the Social Science (SPSS), em que as variáveis foram apresentadas por meio de média e desvio padrão e as categóricas por frequências e porcentagens.

Conforme a resolução 466/12 do Conselho Nacional de Saúde, a pesquisa passou por apreciação ética pelo Comitê de Ética em Pesquisa da Universidade Estadual do Piauí (UESPI), via Plataforma Brasil, com data de aprovação em 26 de Abril de 2018, parecer $n^{\circ}$ 2.624.036, CAAE 84685318.0.0000.5209, e mediante a assinatura de duas vias de um Termo de Consentimento Livre e Esclarecido pelo participante, ficando uma com o mesmo e outra com a pesquisador responsável. Além disso, os pesquisadores se responsabilizaram para garantir a confidencialidade, autonomia, anonimato e não utilização das informações em prejuízo dos sujeitos da pesquisa.

\section{Resultados}

Dos 06 fisioterapeutas, cinco eram do sexo feminino e um do sexo masculino, dos quais apresentavam média de idade igual a 33,16 $\pm 2,91$ anos, com tempo médio de atuação no NASF de 5,6 anos, 05 possuíam pós-graduação, dos quais somente uma fisioterapeuta realizou mestrado em epidemiologia e saúde pública, os demais se especializaram em terapia intensiva. 
Os fisioterapeutas foram questionados se realizam atendimento individual na unidade básica de saúde, e em caso positivo qual a principal demanda, $100 \%$ dos profissionais citaram realizar atendimentos nas unidades básicas de saúde. No qual, as disfunções mais comumente encontradas estão relacionadas às áreas de Traumatoortopedia (100\%), neurologia (83,33\%), reumatologia (50\%) e fisioterapia respiratória (33,33\%).

Em relação a realização de atendimento em domicílio, 100\% dos entrevistados realizam esse tipo de atividade, esses são realizados em conjunto com os integrantes da equipe ou individualmente, e no que diz respeito às principais enfermidades/limitações funcionais que são tratadas nos atendimentos domiciliares individuais, a mais citada foi o Acidente Vascular Encefálico - AVE (100\%), seguido de artrose (33,33\%), pós-operatório de fraturas (33,33\%), sequelados de Traumatismo Crânioencefálio-TCE (33,33\%), e Hanseníase (33,33\%).

No que se refere a realização de atividades voltadas exclusivamente para a saúde da criança e do adolescente, 83,33\% afirmaram realizar atividades em grupo, ações preventivas e de educação em saúde com esse público; $100 \%$ dos entrevistados citaram realizar atividades em grupo, ações preventivas e de educação em saúde voltadas para a saúde da mulher; já em relação à saúde do homem e saúde do idoso 83,33\% dos profissionais citaram a realização de atividades em grupo com esse público, por outro lado o desenvolvimento de ações preventivas e educação em saúde foram citadas por $100 \%$ dos entrevistados.

Quando questionados acerca dos principais procedimentos específicos realizados no atendimento em domicílio, $100 \%$ dos profissionais afirmaram realizar orientações para pacientes e familiares, por outro lado somente $50 \%$ citaram realizar prescrição de exercícios domiciliares, (Gráfico 1).

Gráfico 1. Principais procedimentos específicos realizados nos atendimentos em domicílio pelos fisioterapeutas do NASF-AB de Teresina, PI

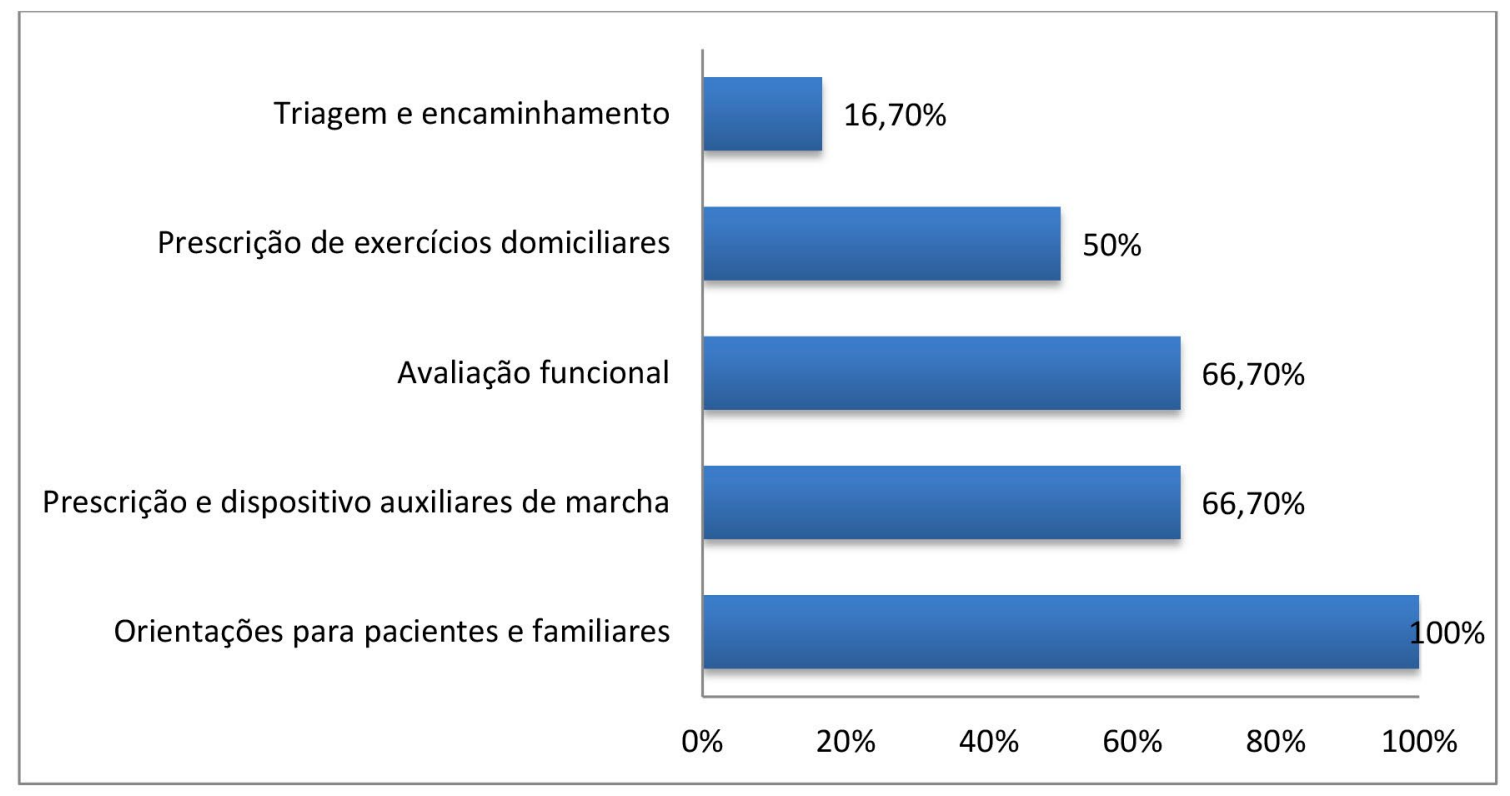

Fonte: Dados da pesquisa, ano 2019

Na última seção do questionário os fisioterapeutas responderam a questões que objetivaram verificar o nível de satisfação desses profissionais com as condições de trabalho, no que diz respeito a infraestrutura e ambiência, bem como o nível de interação entre as equipes, e como resultado obteve-se que a maior parte desses profissionais classificaram como regular as condições de trabalho, e como bom o espaço disponível para atendimento, quando necessário (Gráfico 2). 


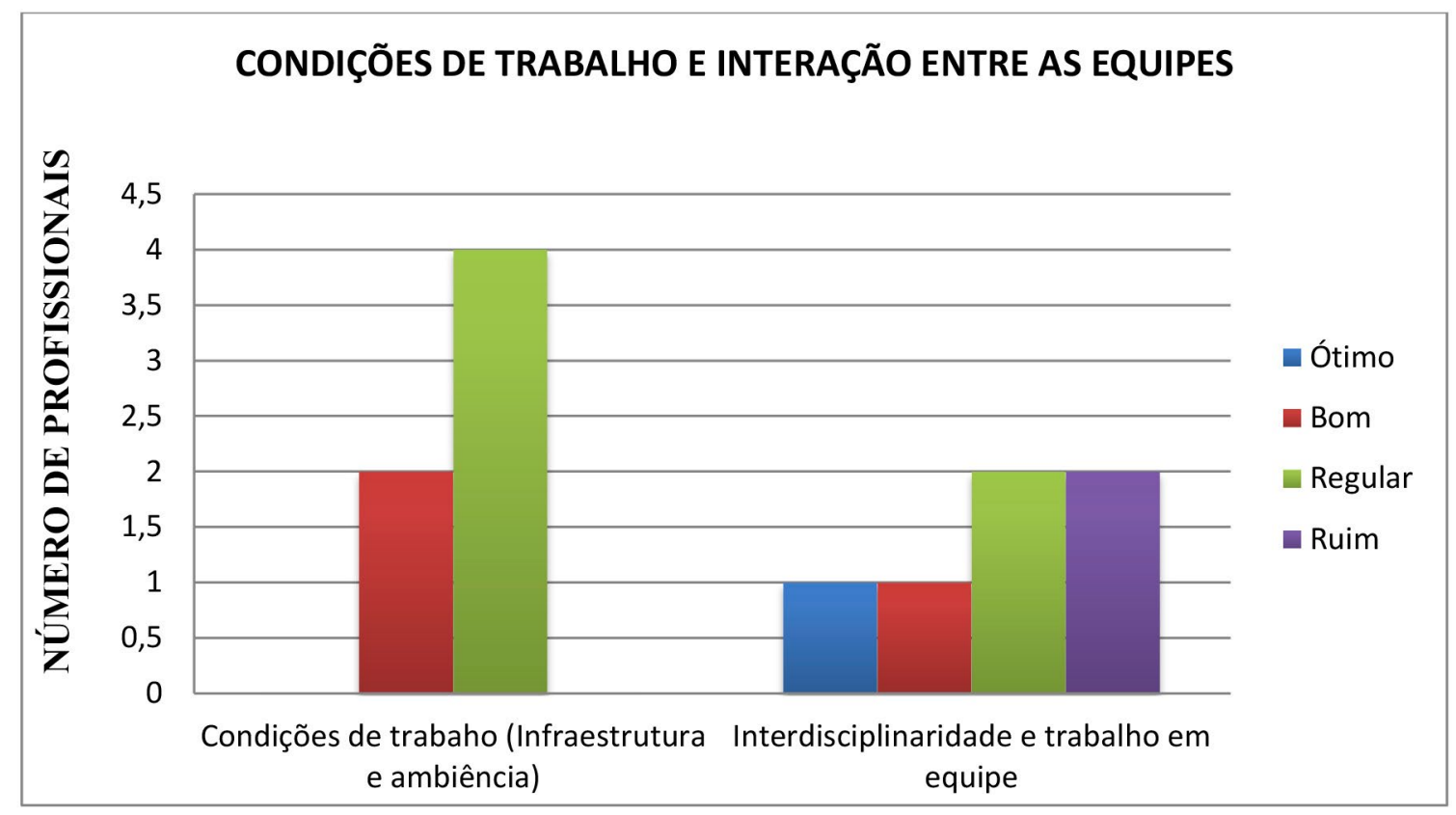

Fonte: Dados da pesquisa, ano 2019.

\section{Discussão}

Os profissionais que compõe o NASF-AB devem nortear sua prática no conceito ampliado de saúde, direcionado a promoção de saúde e prevenção de doenças, por meio de ações compartilhadas com o ESF. Além disso, esses profissionais possuem a potência de contribuição em áreas temáticas específicas, logo também podem atuar diretamente na realização de ações clínicas ou coletivas com os usuários, quando se fizer necessário, de modo integrado e corresponsável ${ }^{6}$.

Nesse sentido, quando questionados acerca da realização de atendimentos individuais na UBS, todos os profissionais afirmaram realizar atendimentos individuais na unidade, e as disfunções mais comuns estão relacionadas às áreas de traumato-ortopedia e neurologia. E muitas dessas queixas e sintomatologias podem ser prevenidas ou atenuadas com a presença do fisioterapeuta nesse nível de atenção. Tais achados estão de acordo com os de Trindade et al. $\frac{15}{}$ que avaliou a prevalência de queixas musculoesqueléticas em adultos de uma unidade básica de saúde da região do Butantã-SP, no qual foi possível verificar nos resultados que este foi o segundo motivo de procura mais frequente, ficando atrás apenas de pedido de laudos/atestados. Além disso, a demanda espontânea por motivos de problemas neurológicos e respiratórios também foram relatados.
Ainda em relação às disfunções mais comuns por áreas de atendimento, Langoni et al. $\frac{16}{}$ também evidenciam que dos usuários de cinco unidades de saúde que realizaram atendimentos individuais com os fisioterapeutas há um predomínio de procura devido a desordens do sistema osteomuscular.

O atendimento em domícilio é uma atividade comum na rotina dos fisioterapeutas que compõem o NASF-AB do munícipio estudado, e de acordo com os resultados desse estudo, o AVE é a enfermidade mais comum nos atendimentos domiciliares. Esses achados corroboram com os dados do estudo de Faria et al. $\stackrel{17}{ }$ que descreveu o perfil do atendimento prestado pelos fisioterapeutas do NASF-AB aos pacientes Pós-AVC, e relatou que os fisioterapeutas foram os membros da equipe que primeiro viram e prestaram cuidados à maioria dos pacientes, e que a maioria dos atendimentos de fisioterapia ocorreu em domicílio, além disso a orientação a pacientes e familiares está entre os serviços mais comuns oferecidos pelos fisioterapeutas.

Outra enfermidade muito comum, sobretudo em idosos, e que necessita da intervenção fisioterapêutica é a artrose, que também foi citada pelos profissionais do presente estudo como uma das enfermidades mais comuns nos atendimentos em domicílio. Esse achado também foi evidenciado no estudo de Silva e 
Sirena ${ }^{18}$, que ao caracterizar o perfil clínico dos usuários da Atenção Primária à Saúde de Porto Alegre-RS encaminhados à fisioterapia, identificou que a osteoartrose está entre as disfunções mais prevalentes nesse público.

Ainda em relação as enfermidades mais comuns nos atendimentos domiciliares citadas pelos fisioterapeutas de Teresina, observa-se uma semelhança com o perfil clínico encontrado na cidade de Porto AlegreRS, conforme mostra os resultados do estudo de Pereira e Gessinger ${ }^{19}$ que dentre as enfermidades citadas como mais prevalentes nesse nível de atenção encontra-se a artrose, pós-operatório de fratura de membros inferiores, e sequelas de AVE, o que enfatiza ainda mais a necessidade do acompanhamento domiciliar contar com a presença do fisioterapeuta.

Dentre as atividades desenvolvidas pelos profissionais entrevistados, há a realização de atendimento em grupo na UBS, e atendimento individual específico. Em relação ao atendimento voltado para a saúde da mulher, todos os profissionais (100\%) afirmaram realizar atendimentos na área descrita, por meio de técnicas fisioterapêuticas e ações preventivas. Em consonância com o estudo de Bispo-Júnior ${ }^{10}$ no que diz respeito a saúde da mulher, um dos grupos beneficiados em relação às orientações posturais é o grupo de gestantes, visto que durante esse período há uma série de alterações, e consequente desconforto para a paciente. Para isso, a atuação da fisioterapia faz-se de suma importância durante essa fase através de orientações posturais, técnicas de alongamento e relaxamento, exercícios que favorecem a circulação, exercícios respiratórios, além do incentivo à amamentação.

No que diz respeito as atividades voltadas para saúde do homem, 83,33\% dos profissionais afirmam realizar atendimentos com esse grupo, porém apontam para a dificuldade da população masculina em aderir às atividades realizadas, sobretudo as preventivas. Esse segmento populacional ainda é pouco trabalhado na atenção básica, uma vez que só procuram os serviços de saúde quando a doença já está instalada ou os que frequentam são os que apresentam uma idade mais avançada, que estão em uma fase da vida em que a saúde tende a se debilitar e torna-los dependentes desse serviço ${ }^{20}$.
Outro grupo abordado foi o da saúde do idoso, e a maioria dos profissionais afirmou realizar atividades em grupo com esse público. Além disso, todos citaram que desenvolvem ações preventivas e educação em saúde. Em consonância com esses achados, o estudo de Aveiro et al. ${ }^{21}$ aponta para a necessidade de atuação do fisioterapeuta voltada ao atendimento de idosos no âmbito da atenção primária, seja na prevenção de agravos e promoção da saúde, quanto para recuperação à saúde. Na prática com esse público é comum a realização de atendimento individual que pode ser na unidade ou domicílio, atividades em grupo na unidade de atenção básica, com práticas de cinesioterapia e atividade física, e orientações.

A fisioterapia na atenção básica é destinada também à saúde da criança e do adolescente, e quando questionados, a maioria dos profissionais confirmou prestar atendimento a esse público tanto no atendimento individual ambulatorial, como em atividades de ação preventiva. Conforme estudo de revisão realizado por David et al.22 observou-se que as enfermidades mais comuns em pacientes pediátricos que necessitam de assistência fisioterapêutica são os casos de afecções respiratórias e motoras. Além disso, também citam complicações referentes ao atraso no desenvolvimento motor infantil. Outra competência do profissional é a atuação em ambientes escolares devido as inúmeras queixas álgicas e alterações posturais de escolares e adolescentes. Essas propostas foram citadas pelos entrevistados do presente estudo como atividades realizadas pelos profissionais na atenção básica.

O atendimento em domicílio foi citado por todos os profissionais entrevistados como uma das práticas realizadas por eles nesse nível de atenção, esse achado se correlaciona favoravelmente com a prática dos fisioterapeutas do Programa de Residência Multiprofissional em Saúde da Família e Comunidade (PRMSFC) da cidade de Fortaleza (CE) que trabalham com a visita domiciliar, pois para eles essa é uma forma de atender as reais demandas da população, uma vez que os profissionais tem um contato direto não só com o paciente, mas com todo o meio familiar que ele está inserido, portanto é possível observar não só condições físicas, mas também problemas sociais e emocionais $\underline{23}$. 
No que tange as principais atividades realizadas pelos fisioterapeutas entrevistados no âmbito da assistência domiciliar destacam-se: orientações para pacientes e familiares (100\%), prescrição de dispositivos auxiliares de marcha $(66,70 \%)$, e prescrição de exercícios domiciliares (50\%). Em consonância com esses achados, o estudo de Pereira e Gessinger ${ }^{19}$ identificou que o perfil dos pacientes que recebem atendimento fisioterapêutico domiciliar, mostrou-se ter um público predominantemente idoso e portadores de doenças crônicas, acompanhados por cuidadores que em sua maioria é um familiar. E dentre as principais atividades desenvolvidas destacam-se as atividades para a melhora da funcionalidade, ações preventivas, orientações a pacientes e seus cuidadores, orientações quanto à realização de exercícios terapêuticos executados pelos cuidadores e adesão da família no processo de tratamento.

Além disso, conforme estudo realizado por VegaRamírez et al..24 as principais técnicas aplicadas no atendimento domiciliar em pacientes com comprometimento motor consistem principalmente em exercícios funcionais, educação de cuidadores e orientações. Dados desse estudo enfatizam ainda que melhorias importantes foram obtidas em termos de capacidade funcional com a atuação da fisioterapia nesse nível de atenção. Esses resultados estão de acordo com a prática realizada pelos fisioterapeutas de Teresina-PI.

Ainda em relação aos procedimentos e atividades realizadas por esses profissionais, conforme achados do presente estudo, a prescrição de dispositivos auxiliares de marcha não é visto como uma dificuldade para os profissionais do NASF-AB de Teresina, uma vez que a maioria $(66,70 \%)$ relatou realizar prescrição desses dispositivos. Contudo, essa realidade é diferente da apontada por fisioterapeutas da atenção básica de um município do interior paulista, que sinalizaram para a dificuldade de prescrição de órteses, mesmo sendo de competência do fisioterapeuta a prescrição de próteses e órteses quando necessário ${ }^{25}$.

A inserção da fisioterapia na atenção primária à saúde tem tido grandes avanços, no entanto uma das limitações para uma atuação mais efetiva são as condições de trabalho, no que diz respeito a infraestrutura e ambiência, visto que quando questionados acerca dessa temática a maioria dos profissionais $(66,66 \%)$ caracterizaram como regular as condições de trabaIho, e em relação a interdisciplinaridade e trabalho em equipe grande parte dos profissionais mostrouse insatisfeito, pois ainda possuem dificuldades relacionadas à articulação entre as equipes. Esses achados encontram apoio em estudos anteriores. Para Amorim et al. $\underline{\underline{26}}$ e Antonio et al. $\underline{25}$ os fisioterapeutas em exercício no NASF-AB apresentam dificuldades para obtenção de espaço físico adequado, falta de recursos tecnológico/equipamentos e falta de interação entre as equipes.

Dentre os possíveis fatores contribuintes para essa insatisfação dos profissionais quanto à falta de interdisciplinaridade e interação entre as equipes, acredita-se que a baixa quantidade de equipes do NASF-AB e o elevado número de equipes do ESF existentes no município estudado seja um dos motivos. Além disso, essa falta de interação entre as equipes pode estar relacionada ao fato de muitos profissionais do ESF desconhecerem as funções dos profissionais do NASF-AB, bem como a real finalidade desse programa, uma vez que tendem a associá-lo à prática clínica e assistencialista, isso contribui para um descompasso e falta de interdisciplinaridade, gerando insatisfação por parte dos profissionais, e consequentemente práticas desarticuladas ${ }^{27}$.

Apesar dos profissionais do NASF-AB não terem como prioridade $\mathrm{o}$ atendimento individual e espaço físico independente, uma vez que não é considerado como porta de entrada do sistema, estes podem utilizar-se do próprio espaço das UBS e do território adstrito para o desenvolvimento das atividades ${ }^{6}$. Entretanto, a falta de material de apoio para o desenvolvimento de atividades lúdicas, somado à falta de local adequado para a execução dessas atividades ainda é um desafio frequente na prática dos profissionais que compõem o NASF-AB, restringindo a atuação desses profissionais e gerando insatisfação ${ }^{28}$.

Vale ressaltar que, em relação ao questionamento acerca das condições de trabalho, por ser uma amostra pequena, pode ser visto como potencial fonte de viés que os participantes poderiam ter receio de revelar o contexto da gestão do trabalho e sua organização e com isso, omitir as reais condições, haja vista que com a publicação do estudo 
seria fácil identificar os informantes, entretanto acredita-se que não houve essa condição, uma vez que em nenhum momento deste estudo os participantes foram identificados, além disso, não houve intimidação por parte dos profissionais em expor a realidade vivenciada, já que estes são funcionários concursados, logo desvelar o contexto da gestão do trabalho não o traria nenhum conflito.

Como citado anteriormente, a baixa quantidade numérica de NASF-AB na cidade de Teresina é um fator que deve ser levado em consideração e colocado como ponto de discussão, visto que essa quantidade é insuficiente para suprir a demanda da população, uma vez que segundo os dados do IBGE (2019) 25 a cidade apresenta população estimada de 864.845 pessoas. Com a pouca quantidade de NASF-AB o acesso do fisioterapeuta para atuação nesse nível de atenção torna-se limitado 29 .

Em virtude disso, é fundamental que haja uma maior discussão acerca desse assunto por parte dos gestores, visto que como evidenciado anteriormente, mostra-se como uma estratégia efetiva para assistência às ESF e consequentemente resolutividade das demandas nesse nível de atenção, ademais investimentos em promoção e prevenção da saúde devem ser tidos como prioridade, sobretudo com os grupos de portadores de doenças crônicas, além do aumento proporcional de idosos na população teresinense que é um dos grupos mais beneficiados com esses serviços.

Diante do exposto, fica evidente a necessidade de ampliação do NASF-AB na cidade de Teresina, bem como maiores investimentos em materiais para o desenvolvimento de atividades com os grupos comunitários, investimentos em educação permanente e educação continuada para os profissionais já inseridos, e aumento do número de residências multiprofissionais no Estado do Piauí.

\section{Conclusão}

O presente estudo permitiu concluir que os fisioterapeutas que atuam no NASF-AB de Teresina-PI realizam atendimentos tanto individual, como de forma coletiva e esses atendimentos ocorrem tanto na UBS como em domicílio, a depender da demanda, condição física dos pacientes e dos recursos disponíveis.
Em relação aos atendimentos de forma coletiva, as atividades são realizadas tanto em ambientes fora da UBS, como na própria unidade, e dentre os grupos existentes o de maior adesão são os voltados à saúde da mulher e saúde do idoso.

Os atendimentos domiciliares são realizados em conjunto com os integrantes da equipe ou individualmente, dentre as atividades realizadas por esse profissional no ambiente domiciliar destacam-se orientações para pacientes e familiares, prescrição de dispositivos auxiliares de marcha e prescrição de exercícios domiciliares. Já em relação a realização de atividades em grupo, durante essas ações são discutidas temáticas variadas, de acordo com o perfil de cada grupo.

Além disso, observou-se que mesmo no nível de atenção básica, esses profissionais ainda possuem uma tendência a relacionarem suas atividades à prática clinica e assistencialista. Possivelmente a formação do profissional tenha influência sobre esse resultado, visto que somente um fisioterapeuta tem formação ligada à saúde pública, os demais focaram na formação em outras especialidades. Além disso, os profissionais apontam a falta de interdisciplinaridade e ações compartilhadas com outros profissionais, sendo que essa é uma das principais características do trabalho no NASF-AB, o apoio matricial.

Faz-se necessário a realização de mais estudos em outros Estados e municípios brasileiros a fim de conhecer a realidade de atuação desses profissionais nas diversas regiões do país, e assim melhor delinear seu papel nesse nível de atenção.

\section{Financiamento}

O estudo foi financiado pela Fundação de Amparo à Pesquisa do Estado do Piauí (FAPEPI) em parceria com a Universidade Estadual do Piauí (UESPI).

\section{Contribuições dos autores}

Silva AD participou da concepção, delineamento, busca e análise estatística dos dados da pesquisa, interpretação dos resultados, levantamento bibliográfico e redação do artigo científico. Nogueira LT orientou o estudo, participou do delineamento e redação do artigo científico. Silva HGN Participou da interpretação dos resultados e análise estatística. Frota SCM Participou do levantamento bibliográfico. Todos os autores participaram da revisão crítica e aprovação final do manuscrito. 


\section{Conflitos de interesses}

Nenhum conflito financeiro, legal ou político envolvendo terceiros (governo, empresas e fundações privadas, etc.) foi declarado para nenhum aspecto do trabalho submetido (incluindo, mas não se limitando a subvenções e financiamentos, participação em conselho consultivo, desenho de estudo, preparação de manuscrito, análise estatística, etc.).

\section{Referências}

1. Ministério da Saúde. Gabinete do Ministro. Portaria n 648/ GM de 28 de março de 2006. Aprova a Política Nacional de Atenção Básica, estabelecendo a revisão de diretrizes e normas para a organização da Atenção Básica para o Programa de Saúde da Família (PSF) e o Programa Agentes Comunitários de Saúde (PACS). Diário Oficial da União, Brasília, DF, 2006.

2. Fernandes SCS, Ros MA. Desconstruir para transformar: o perfil do fisioterapeuta para o Núcleo de Apoio à Saúde da Família. Fisioter Bras. 2018;19(2):249-258. doi: 10.33233/fb.v19i2.867

3. Braghini CC, Ferretti F, Ferraz L. The role of physical therapists in the context of family health support centers. Fisioter Mov. 2017;30(4):703-713. doi: 10.1590/1980-5918.030.004.a005

4. Ministério da Saúde. Portaria GM n 154, de 24 de janeiro de 2008: Cria os Núcleos de Apoio à Saúde da Família, Nasf. Diário Oficial da União, Brasília, DF, 4 mar.

5. Ministério da Saúde. Portaria $n^{\circ} 2.436$, de 21 de setembro de 2017. Aprova a Política Nacional de Atenção Básica, estabelecendo a revisão de diretrizes para a organização da Atenção Básica, no âmbito do Sistema Único de Saúde (SUS). Diário Oficial da República Federativa do Brasil, Poder Executivo, Brasília, DF: Ministério da Saúde, 2017.

6. Ministério da Saúde. Secretaria de Atenção à Saúde. Departamento de Atenção Básica. Núcleo de Apoio à Saúde da Família-Volume 1: Ferramentas para a gestão e para o trabalho cotidiano. Brasília, DF: Ministério da Saúde, 2014. 116 p.

7. Souza MC, Bomfim AS, Souza JN, Franco TB. Fisioterapia e Núcleo de Apoio à Saúde da Família: conhecimento, ferramentas e desafios. Mundo saúde. São Paulo, 2013; 37(2):176-184. doi: 10.15343/0104-7809.2013372176184

8. Alves NS, Portela ERM, Gonçalves FS, Guimarães TS, Alencar AJF, Mendes ES et al. Perspectivas sobre o trabalho do fisioterapeuta na atenção básica: uma revisão integrativa. Revista CPAQV. 2020;12(1). doi: 10.36692/cpaqv-v12n1-28

9. Souza MC, Rocha ÂA, Cabral T, Souza JN. Fisioterapia, Acesso e Necessidades de Saúde: limites e possibilidades na atenção básica. Rev Pesqui Fisioter. 2015;5(2). doi: 10.17267/2238-2704rpf. v5i2.499
10. Bispo-Júnior JP. Fisioterapia e saúde coletiva: desafios e novas responsabilidades profissionais. Ciênc Saúde Colet. 2010;15(Supl. 1):1627-1636. doi: $10.1590 / s 1413-81232010000700074$

11. Nascimento AG, Cordeiro JC. Núcleo Ampliado de Saúde da Família e Atenção Básica: análise do processo de trabalho. Trab Educ Saúde. 2019;17(2). doi: 10.1590/1981-7746-sol00194

12. Conselho Municipal de Saúde de Teresina (CMS). Plano Municipal de Saúde 2018-2021. [Internet] 2018. [acesso em 2020 ago 18] Disponível em: https://semplan.teresina.pi.gov.br/wpcontent/uploads/sites/39/2018/05/PMS-2018-2021-Vers\%C3\%A3ofinal.pdf

13. Cândido AM. Atuação da fisioterapia nos Núcleos de Apoio à Saúde da Família: um estudo no município de Campina Grande - PB. [monografia]. Campina Grande: Universidade Estadual da Paraíba; 2015.

14. Sousa KRM. Conhecimento dos acadêmicos de Fisioterapia na APS e a realidade de atuação dos fisioterapeutas inseridos no NASF de Campina Grande-PB [monografia]. Paraíba: Universidade Estadual da Paraíba; 2016.

15. Trindade KMC, Schmitt ACB, Casarotto RA. Queixas musculoesqueléticas em uma Unidade Básica de Saúde: implicações para o planejamento das ações em saúde e fisioterapia. Fisioter Pesqui. 2013;20(3):228-234. doi: 10.1590/ S1809-29502013000300006

16. Langoni CS, Valmorbida LA, Resende TL. A introdução de atendimentos por fisioterapeutas em unidades da atenção primária em saúde. Rev Bras Promoç Saúde. 2012;25(3):261-270. doi: 10.5020/18061230.2012.p261

17. Faria CDCM, Araújo DC, Carvalho-Pinto BPB. Assistência do fisioterapeuta da atenção primária a indivíduos pós-acidente vascular cerebral. Fisioter Mov. 2017;30(3):527-536. doi: 10.1590/1980-5918.030.003.ao11

18. Silva GG, Sirena SA. Perfil de encaminhamentos a fisioterapia por um serviço de Atenção Primária à Saúde, 2012. Epidemiol Serv Saúde. 2015;24(1):123-133. doi: 10.5123/s167949742015000100014

19. Pereira BM, Gessinger CF. Visão da equipe multidisciplinar sobre a atuação da fisioterapia em um programa de atendimento domiciliar público. Mundo Saúde. 2014;38(2):210-218. doi: $\underline{10.15343 / 0104-7809.20143802210218}$

20. Oliveira MM, Daher DV, Silva JLL, Andrade SSCA. A saúde do homem em questão: busca por atendimento na atenção básica de saúde. Ciênc Saúde Colet. 2015;20(1):273-278. doi: 10.1590/1413-81232014201.21732013

21. Aveiro MC, Aciole GG, Driusso P, Oishi J. Perspectivas da participação do fisioterapeuta no Programa Saúde da Família na atenção à saúde do idoso. Ciênc Saúde Colet. 2011;16(Supl.1):1467-1478. doi: 10.1590/s1413$\underline{81232011000700082}$ 
22. David MLO, Ribeiro MÂGDO, Zanolli MDL, Mendes RT, Assumpção MSD, Schivinski CIS. Proposta de atuação da fisioterapia na saúde da criança e do adolescente: uma necessidade na atenção básica. Saúde Debate. 2013;37(96):120129. doi: $10.1590 / s 0103-11042013000100014$

23. Bezerra MIC, Lima MJMR, Lima YCP. A visita domiciliar como ferramenta de cuidado da fisioterapia na estratégia saúde da família. Sanare. 2015;14(1):76-80.

24. Vega-Ramírez FA, López-Liria R, Granados-Gámez G, Aguilar-Parra JM, Padilla-Góngora D. Analysis of home-based rehabilitation in patients with motor impairment in primary care: a prospective observational study. BMC Geriatr. 2017;17(1):145. doi: 10.1186/s12877-017-0526-0

25. Antoni ACFT, Tonhom SFR, Chirelli MQ. Cuidado ao idoso na atenção básica: práticas de educação em saúde do fisioterapeuta. Rev Bras Promoç Saúde. 2016;29(Supl):5-15. doi: 10.5020/18061230.2016.sup.p5
26. Amorim JF, Ferreira JB, Souza LCS, Carvalho TS, Morais KCS. Percepção dos Fisioterapeutas sobre sua Atuação no Núcleo de Apoio à Saúde da Família. Revista InterScientia. 2017;5(1):105-115.

27. Souza TS, Medina, MG. Nasf: fragmentação ou integração do trabalho em saúde na APS?. Saúde Debate. 2018;42(spe2):145158. doi: $\underline{10.1590 / 0103-11042018 s 210}$

28. Araujo Neto JD, Albuquerque IMAN, Lira GV, Bosi MLM. Aspectos restritivos à integralidade nos Núcleos de Apoio à Saúde da Família: o olhar dos stakeholders. Physis. 2019;28(4):e280417. doi: $10.1590 /$ s0103-73312018280417

29. Instituto Brasileiro de Geografia e Estatística. Panorama Teresina [Internet]. 2019. [acesso em 2020 ago 18]. Disponível em: https://cidades.ibge.gov.br/brasil/pi/teresina/panorama 